\title{
Amplitude and phase effects on the synchronization of delay-coupled oscillators
}

\author{
O. D'Huys, ${ }^{1, a)}$ R. Vicente, ${ }^{2}$ J. Danckaert, ${ }^{1,3}$ and I. Fischer ${ }^{4}$ \\ ${ }^{1}$ Department of Physics (DNTK), Vrije Universiteit Brussel, Pleinlaan 2, 1050 Brussel, Belgium \\ ${ }^{2}$ Max-Planck-Institute for Brain Research, Deutschordenstraße 46, 60528 Frankfurt am Main, Germany and \\ Frankfurt Institute for Advanced Studies (FIAS), Ruth-Moufang-Straße 1, 60438 Frankfurt, Germany \\ ${ }^{3}$ Department of Applied Physics and Photonics (TONA), Vrije Universiteit Brussel, Pleinlaan 2, 1050 Brussel, \\ Belgium \\ ${ }^{4}$ Instituto de Fisica Interdisciplinar y Sistemas Complejos, IFISC (UIB-CSIC), Campus Universitat de les \\ Illes Balears, E-07122 Palma de Mallorca, Spain
}

(Received 24 September 2010; accepted 1 November 2010; published online 3 December 2010)

\begin{abstract}
We consider the behavior of Stuart-Landau oscillators as generic limit-cycle oscillators when they are interacting with delay. We investigate the role of amplitude and phase instabilities in producing symmetry-breaking/restoring transitions. Using analytical and numerical methods we compare the dynamics of one oscillator with delayed feedback, two oscillators mutually coupled with delay, and two delay-coupled elements with self-feedback. Taking only the phase dynamics into account, no chaotic dynamics is observed, and the stability of the identical synchronization solution is the same in each of the three studied networks of delay-coupled elements. When allowing for a variable oscillation amplitude, the delay can induce amplitude instabilities. We provide analytical proof that, in case of two mutually coupled elements, the onset of an amplitude instability always results in antiphase oscillations, leading to a leader-laggard behavior in the chaotic regime. Adding selffeedback with the same strength and delay as the coupling stabilizes the system in the transverse direction and, thus, promotes the onset of identically synchronized behavior. (C) 2010 American Institute of Physics. [doi:10.1063/1.3518363]
\end{abstract}

Synchronization phenomena of interacting elements are omnipresent in nature. A coupling delay may naturally appear in the interaction between the nodes, as the propagation time of a signal. Such a delayed coupling can modify dynamics drastically, giving rise to complex behavior and different synchronization patterns. The coupling topology plays an essential role in the onset of these patterns. For example, when coupling two elements with delay, only achronal synchronization is observed in different systems as semiconductor lasers, neuronal models, or electronical circuits. Zero-lag synchronization can be restored when coupling the two elements through a relay. We study analytically and numerically the synchronization properties of two generic oscillators coupled with delay: the Kuramoto oscillator, which only describes the phase dynamics, and the Stuart-Landau oscillator, which includes a variable amplitude. We first review the influence of a delayed feedback in both models. Then we study in detail the origin of achronal synchronization and the effect of a relay.

\section{INTRODUCTION}

Synchronization phenomena, meaning the adjustment of rhythms between interacting elements, occur in different physical systems with different space and time scales. ${ }^{1,2}$ Synchronized behavior has been observed in brain activity, ${ }^{3,4}$

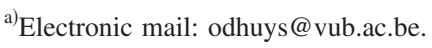

coupled lasers, ${ }^{5,6}$ Josephson junctions, ${ }^{7,8}$ and social behavior, ${ }^{9}$ to name only a few examples.

Synchronization occurs due to coupling between the individual elements. This coupling is not necessarily instantaneous and might exhibit propagation delays which are comparable to or larger than the characteristic oscillatory time scale of the individual oscillators. The influence of such a latency in the coupling has already been addressed for many years in systems such as molecular oscillators, ${ }^{10}$ electronic circuits, ${ }^{11}$ Van der Pol oscillators, ${ }^{12}$ traffic models, ${ }^{13}$ optically and optoelectronically coupled semiconductor lasers, and in generic model systems such as phase oscillators ${ }^{14}$ or logistic maps. ${ }^{15}$ The early investigations could, however, only address the situations of small delays and weak nonlinearity. However, both new analytical ${ }^{16}$ and numerical tools ${ }^{17,18}$ have been developed for delay differential equation problems, facilitating the recent multidisciplinary activities on the delayed synchronization dynamics. 19,20

A coupling delay can induce complex behavior in a network, and the nodes organize in different synchronization patterns. The coupling configuration plays a crucial role in the synchronization behavior. When, for instance, two lasers are coupled face to face, the lasers behave chaotically due to the delay. Only generalized synchronization of leaderlaggard type is observed, although the setup is completely symmetric. $^{21,22}$ The isochronally synchronized solution also exists, but it was found to be unstable to small perturbations. ${ }^{23,24}$ Also in an optoelectronic setup the two lasers do not synchronize identically. ${ }^{25}$ The same experiment 
has been performed with delay-coupled chaotic electronic circuits, and again this generalized synchronization of leaderlaggard type was observed. ${ }^{26}$ The origin of this dynamical behavior is, to the best of our knowledge, not fully understood. On the other hand, complete synchronization between lasers, without a time lag, has been observed when a semitransparent mirror or a third laser was placed in between the elements. ${ }^{27-29}$ Such a configuration with a relay seems to be favorable to stabilize isochronal synchronization, not only for the laser system but also for other systems coupled with delay such as Hodgkin-Huxley or integrate-and-fire neurons ${ }^{30}$ or chaotic electronic circuits. ${ }^{26}$

In this manuscript, we consider generic classes of delaycoupled systems and study the isochronal and generalized synchronization solutions and the transitions between them. More specifically, we consider three different delay-coupled configurations of oscillators and analyze their dynamics. For each network, we compare two models of different complexities, one only describing the phase of an oscillator (Kuramoto) and one allowing for variable phase and amplitude [Stuart-Landau (SL)]. In this way, we can identify which properties lead to achronal synchrony in delaycoupled elements.

The article is organized as follows. In Sec. II we introduce the two oscillator models and their motivation. Then, we describe analytically the dynamics of a single oscillator with delayed feedback in Sec. III. We proceed in Sec. IV with two mutually coupled elements, where we analyze in detail the origin of the antiphase oscillations leading to leader-laggard dynamics. In Sec. V we consider the addition of feedback to the network, which is shown to stabilize the zero-lag synchronized solutions. We conclude by discussing our main results and giving an outlook of future work on delay-coupled systems.

\section{HIERARCHY OF OSCILLATOR MODELS}

The simplest approach to describe an oscillating system is by its oscillation phase. Therefore, as a first class of models we consider delay-coupled Kuramoto oscillators, which describe the dynamics of each element by a single phase variable. Interestingly, many types of oscillators can be reduced to Kuramoto models in the weak coupling regime. ${ }^{31,32}$ This model is given by

$$
\dot{\phi}_{n}=\omega_{0}+\kappa \sum A_{m n} \sin \left(\phi_{m}(t-\tau)-\phi_{n}(t)+\theta\right),
$$

where $\omega_{0}$ is the natural frequency of the oscillators, $\kappa$ stands for the coupling strength, $\theta$ is a coupling phase, and $\tau$ is the coupling delay. The adjacency matrix $A$ describes the topology of the network.

As a second model we consider Stuart-Landau (SL) oscillators. Stuart-Landau is a generic model for weakly nonlinear oscillators and limit-cycles close to a Hopf bifurcation. For example, the van der Pol oscillator is a particular case [for $\beta=0$ in Eq. (2)] of Stuart-Landau. ${ }^{33}$ Furthermore, when taking the appropriate limits for a continuum description SL derives into the famous Ginzburg-Landau equation. A Stuart-Landau oscillator is described by a phase and an amplitude according to

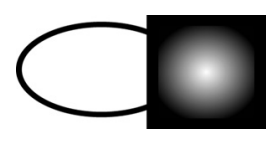

FIG. 1. Schematic drawing of an oscillator with delayed feedback

$$
\dot{a}_{n}=a_{n}\left(1-\left|a_{n}\right|^{2}\right)+i \beta a_{n}\left|a_{n}\right|+\kappa e^{i \theta} \sum A_{m n} a_{m}(t-\tau),
$$

where $\beta$ represents a coupling between amplitude and phase, while the rest of parameters have a similar meaning as in the Kuramoto description. Notice, however, that the dependent variable $a$ is a complex variable.

For both models we consider the case of direct coupling, i.e., oscillators are coupled by the delayed variable of a coupled element and not diffusively by the difference between them. The diffusively coupled and the directly coupled systems can sometimes (but not always) be mapped onto each other. They show similar dynamics in the presence of a coupling delay, one of the main differences is that amplitude death of identical elements can occur when the coupling is diffusive $^{34,35}$ and not when it is direct.

Networks of diffusively coupled Stuart-Landau oscillators have recently been studied by Choe et al. ${ }^{36}$ In this work a master stability function for delay-coupled systems is introduced, which provides a way to decouple network and node dynamics and to easily compare the synchronization properties of networks numerically. This work concentrates on the stability of constant amplitude solutions in relation to the coupling phase $\theta$, while we are mainly interested in the dynamics when the amplitude is no longer stable, i.e., the oscillation pattern of the amplitude and the chaotic synchronization pattern.

A Stuart-Landau oscillator is also closely related to the equations for coupled semiconductor lasers. Omitting the term linear in $a_{n}$, we obtain a reduced version of the LangKobayashi equations, ${ }^{37}$ valid for weak coupling, long delays, and low pump currents of semiconductor lasers. The parameter $\beta$ can then be related to the linewidth enhancement factor. Both models exhibit qualitatively very similar dynamics when coupled with delay. Lasers are usually coupled directly as well, which makes the comparison between our model and the laser system easier.

\section{ONE OSCILLATOR WITH FEEDBACK}

In this section we review the most basic motif with delay (shown in Fig. 1), being one oscillator with delayed feedback. ${ }^{37}$ We start by only considering the phase dynamics and briefly commenting on the stability of frequency locked solutions. In the second part of this section we take into account amplitude effects by studying the full Stuart-Landau equation with feedback. There, a stability analysis for the long delay limit is presented together with numerical simulations to track the route to chaos in the system.

The system is modeled by

$$
\dot{a}=a\left(1-|a|^{2}\right)+i \beta a|a|^{2}+\kappa e^{i \theta} a(t-\tau) .
$$




\section{A. A phase oscillator with delayed feedback}

Assuming a fixed amplitude and taking only the phase dynamics into account, one can reduce Eq. (3) to a corresponding Kuramoto equation for the phase

$$
\dot{\phi}=\beta+\kappa \sqrt{1+\beta^{2}} \sin (\phi(t-\tau)-\phi+\theta+\arctan \beta) .
$$

An important class of solutions have a fixed frequency of oscillation $\phi(t)=\omega t$; we will call them modes in the following. Imposing solutions of such class in the former equation implies that $\omega$ satisfies the following transcendental equation:

$$
\omega=\beta+\kappa \sqrt{1+\beta^{2}} \sin (\theta+\arctan \beta-\omega \tau) .
$$

When performing a linear stability analysis, similar as in Refs. 38 and 39, we find that the solutions for which

$$
\cos (\theta+\arctan \beta-\omega \tau)>0
$$

are stable foci. Such modes are stable for any value of the delay.

On the other hand, if

$$
\kappa \tau \sqrt{1+\beta^{2}} \cos (\theta+\arctan \beta-\omega \tau)<-1,
$$

holds, the solution is a saddle point, often referred as antimode. Unstable solutions are hence only possible for sufficiently strong feedback strength or sufficiently long delay times.

\section{B. A Stuart-Landau oscillator with delayed feedback}

If we consider the full system of Eq. (3), a solution with frequency $\omega$ defined by Eq. (5) corresponds to a state with fixed amplitude and frequency $a(t)=r e^{i \omega t}$, where the amplitude can be determined as

$$
r^{2}=1+\kappa \cos (\theta-\omega \tau) .
$$

One can demonstrate, from Eqs. (5) and (8), that these modes lie on an ellipse in the $\left(r^{2}, \omega\right)$-plane. Furthermore, foci and saddles occupy different halves of an ellipse. One must note that the solutions for which $r^{2}<0$ holds are unphysical.

\section{Stability analysis}

The stability of the modes in the full system is determined by the solutions of the characteristic equation which reads

$$
\begin{aligned}
\left(\lambda+r^{2}\right. & \left.+\kappa\left(1-e^{-\lambda \tau}\right) \cos (\theta-\omega \tau)\right)^{2} \\
= & r^{4}+2 \beta \kappa r^{2} \sin (\theta-\omega \tau)\left(1-e^{-\lambda \tau}\right) \\
& -\kappa^{2} \sin ^{2}(\theta-\omega \tau)\left(1-e^{-\lambda \tau}\right)^{2} .
\end{aligned}
$$

In lowest order of the feedback strength $\kappa$, one can find approximately the same stability properties as in Kuramoto system (6). Neglecting the feedback delay time, the system has eigenvalues $\lambda=-2 r^{2}$ (corresponding to an stable amplitude) and $\lambda=0$ (associated with the invariance under a phase shift). It can be shown that for delay times short enough the system still remains fully stable. However, for long delays the foci as we shall see can loose stability in a Hopf bifurcation, which ultimately leads to the development of chaotic amplitude dynamics.

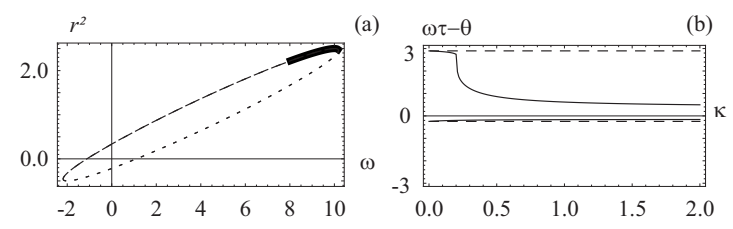

FIG. 2. Stability analysis in the long delay limit. (a) The constant amplitude solutions for one oscillator with feedback [Eq. (3)] are plotted in the $\left(r^{2}, \omega\right)$-plane. The continuous thick line of the ellipse contains stable foci, unstable foci are located in the dashed part, and the dotted line covers saddle points. Parameters are $\kappa=2$ and $\beta=4$. (b) The stable part of the ellipse, parametrized by $\omega \tau-\theta$, is plotted vs the feedback strength $\kappa$. The values of $\omega \tau-\theta$ between the continuous lines correspond to stable foci. Note that for $\omega \tau-\theta=0$, which stands at the top of the ellipse, the solution is always stable. The dashed lines indicate the Kuramoto stability boundaries. $\beta=4$.

The stability properties of periodic solutions (modes) in generic systems with long delayed feedback have been studied by Yanchuk et al. ${ }^{40,41}$ Here, we perform the analysis specifically for a Stuart-Landau oscillator. This will allow us to compare the properties of feedback systems to the mutually coupled configuration described in Sec. IV.

We proceed then to determine which modes are stable in the long delay limit. We assume $\lambda \tau=O(1)$ in Eq. (9) and expand

$$
\lambda=\frac{\lambda_{1}}{\tau}+\frac{\lambda_{2}}{\tau^{2}}+\frac{\lambda_{3}}{\tau^{3}}+O\left(\tau^{-4}\right) .
$$

In zeroth order we obtain as a solution

$$
\lambda_{1}=2 k \pi i,
$$

an eigenvalue which corresponds to an oscillation period of the amplitude equal to the delay time.

Additionally, we find a real root, with $\lambda_{1}<0$ for the upper half of the ellipse and a $\lambda_{1}>0$ for the lower half. Therefore, the modes located on the lower half of the ellipse are saddles, just like the corresponding solutions for the phase oscillator.

In first order of $\tau^{-1}$ we obtain a correction to the oscillation period $P$ as follows:

$$
P \approx \tau+\frac{1}{\kappa \sqrt{1+\beta^{2}} \cos (\arctan \beta-\omega \tau+\theta)} .
$$

The stability of the solutions in the upper half of the ellipse is determined by the third order term. More precisely, the stability of a mode is shown to depend on its position on the ellipse as parametrized by $\omega \tau$. Figure 2(a) shows the part of the ellipse containing stable modes as a function of the feedback strength. The top of the ellipse $(\theta-\omega \tau=0)$, which contains the modes of maximal amplitude, is always stable for all values of the feedback strength. For small feedback strength, the borders of stability of the modes are close to the boundaries obtained for Kuramoto, as displayed in Fig. 2(b). We note that an analysis of the stability of the modes with a pseudocontinuous spectrum, as it has been developed by Yanchuk et al., ${ }^{41,42}$ leads to the same stability properties.

Thus, in the long delay limit, we expect from Fig. 2 stable amplitude behavior for weak feedback and the devel- 


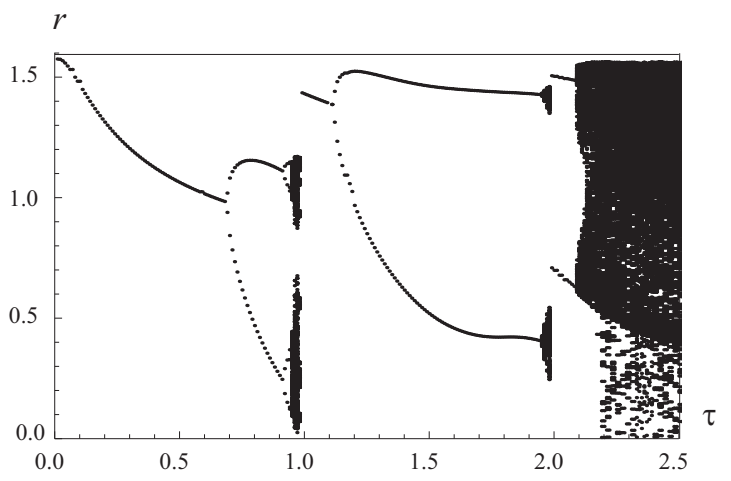

FIG. 3. The bifurcation diagram for a Stuart-Landau oscillator with delayed feedback, described by Eq. (3). Parameters: $\beta=4, \kappa=1.5$, and $\theta=0$.

opment of periodic or chaotic dynamics coexisting with some stable large-amplitude modes for stronger feedback regimes.

\section{Emergence of chaos}

To study the dynamics for moderate feedback strength, when most of the foci are unstable, we numerically simulated a Stuart-Landau oscillator with delayed feedback. As bifurcation parameter we choose the feedback round-trip time $\tau$. Our integration code is based on a Heun algorithm adapted to delayed differential equations. Since the delay generates multiple modes, multistability is always present in such a system, and different initial conditions can lead to different dynamical behaviors. To better compare the dynamics across different delay values, we have used the same initial conditions for each simulation, corresponding to the oscillator's dynamics without feedback $(r=1$ and $\omega=\beta)$. In any case, when the dynamics is chaotic (and involves more than one mode), the qualitative behavior of the oscillator depends less on the initial conditions than for more regular regimes.

The emergence of chaos is shown in Fig. 3 where we display the bifurcation diagram of the amplitude of oscillation as the delay time increases.

For small feedback delays, the oscillator reaches a stable mode. For such short delays, the constant amplitude of oscillation $r$ decreases as the delay increments. At a certain delay $(\tau \approx 0.7)$ this mode undergoes a Hopf bifurcation and oscillations (of the amplitude) around this mode are observed. The time series of the amplitude and the trajectory in the amplitude-frequency space are plotted in Figs. 4(a) and 4(b). As the delay increases further, the period doubles [Figs. 4(c) and 4(d)] and aperiodic oscillations around this mode [shown in Figs. 4(e) and 4(f)] emerge. By increasing the delay even further, the system reaches a stable mode again, which undergoes a first and second Hopf bifurcation on the route to chaos. For longer feedback times, the chaotic dynamics may involve more modes and saddle points, as illustrated in Figs. 4(g) and 4(h).

Many of the results here obtained find analogous in the Lang-Kobayashi description of lasers with feedback. ${ }^{43}$ For example, the external cavity modes of a laser also arrange on an ellipse in the optical intensity-frequency plane. Moreover, the stability of the maximum amplitude solution that we de-

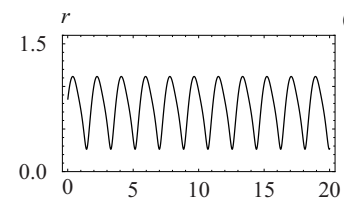

(a)
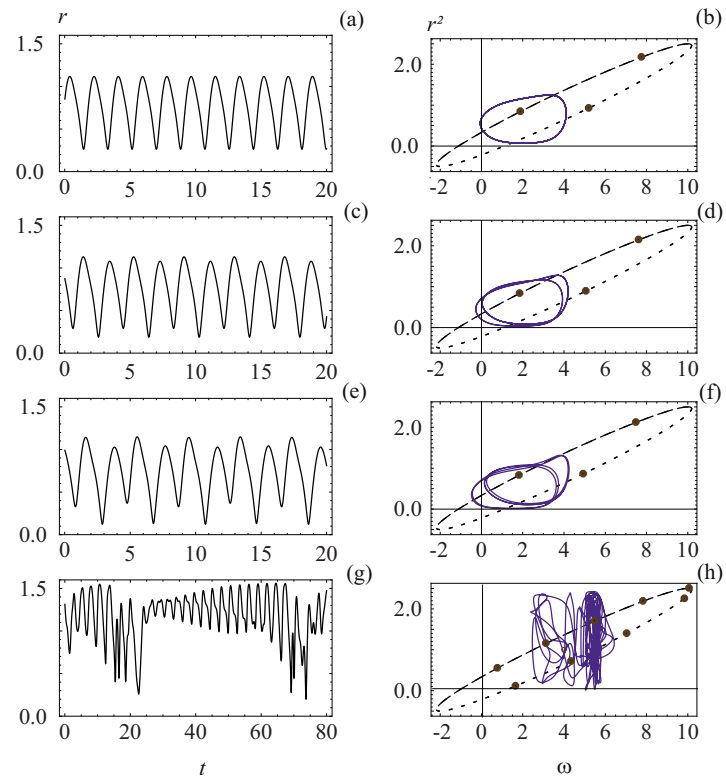

FIG. 4. (Color online) Amplitude dynamics (left) and projection of the dynamics on the $\left(r^{2}, \omega\right)$-space (right) in dependence of the delay time $\tau$. For the right side panels, the $x$-axis represents $(\phi(t)-\phi(t-\tau)) / \tau \approx \omega$. In each right side panel the black dots locate the stable amplitude solutions. The upper part of the ellipse contains the modes stable in the phase oscillator limit and is plotted with a long dashed line, the lower part (short dashed) contains the saddle solutions. Parameters are fixed to $\beta=4, \kappa=1.5, \theta=0$. The delay for each panel is $\tau=0.9[(\mathrm{a})$ and (b)], $\tau=0.92$ [(c) and (d)], $\tau=0.94$ [(e) and (f)], and $\tau=2.5[(\mathrm{~g})$ and $(\mathrm{h})]$.

scribed seems to correspond to that of the highest gain mode for a laser subject to delayed feedback. ${ }^{44}$ The route to chaos of a Stuart-Landau oscillator with feedback is also related to the one found in semiconductor lasers. ${ }^{45-47}$

\section{TWO MUTUALLY COUPLED ELEMENTS}

In the following we consider the case of two oscillators mutually coupled with delay. A schematic representation is shown in Fig. 5. This configuration is modeled by the system

$$
\begin{aligned}
& \dot{a}_{1}=a_{1}\left(1-\left|a_{1}\right|^{2}\right)+i \beta a_{1}\left|a_{1}\right|^{2}+\kappa e^{i \theta} a_{2}(t-\tau), \\
& \dot{a}_{2}=a_{2}\left(1-\left|a_{2}\right|^{2}\right)+i \beta a_{2}\left|a_{2}\right|^{2}+\kappa e^{i \theta} a_{1}(t-\tau) .
\end{aligned}
$$

Since we assume the two oscillators to be identical, the configuration is completely symmetric. Therefore, the identically synchronized solution exists. In the case of synchronized behavior, the dynamics corresponds to that of one oscillator with delayed feedback. The question hence arises whether, and in which regime this solution is stable.

For the corresponding phase oscillator network, this problem can easily be solved analytically. The system allows modes corresponding to in-phase $\left(\phi_{1}(t)=\phi_{2}(t)\right)$ and antiphase $\left(\phi_{1}(t)=\phi_{2}(t)+\pi\right)$ synchronized solutions. In-phase states are stable if

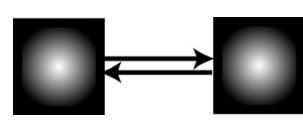

FIG. 5. Schematic representation of two mutually coupled oscillators 


$$
\kappa \cos (\theta+\arctan \beta-\omega \tau)>0
$$

and antiphase states if

$$
\kappa \cos (\theta+\arctan \beta-\omega \tau)<0,
$$

without any further restriction on the coupling strength or delay time. ${ }^{38,39,48}$ There is no discrepancy between the inphase and the antiphase solutions, in the sense that their stability properties are very similar. For long delay times, the stability properties of the in-phase state are the same for one oscillator with feedback and for two mutually coupled oscillators. For short delay times or without delay, saddle point solutions are also possible when we consider two oscillators.

\section{A. Parallel and transverse directions}

In the full system, we can also distinguish between inphase modes $a_{1}(t)=a_{2}(t)=r e^{i \omega t}$ and antiphase modes $a_{1}(t)=$ $-a_{2}(t)=r e^{i \omega t}$. Note that under the transformation $\theta \rightarrow \theta+\pi$, in- and antiphase modes map onto each other, just like for the phase oscillators. We shall not consider possible asymmetric modes here, where the oscillators have a different amplitude but the same frequency.

In the following analysis we distinguish a parallel direction $\left(a_{1}+a_{2}\right) / 2$, which describes the dynamics inside the synchronization manifold and a transverse direction $\left(a_{1}-a_{2}\right) / 2$. In other networks this approach would correspond to applying a master stability function. ${ }^{36}$ In the parallel direction the dynamics (and stability) is the same as for one oscillator with feedback described in Sec. III. A Hopf instability in this direction leads to in-phase oscillations of the amplitude.

For the transverse direction, we obtain the characteristic equation,

$$
\begin{aligned}
\left(\lambda+r^{2}+\kappa\left(1+e^{-\lambda \tau}\right) \cos (\theta-\omega \tau)\right)^{2} \\
=r^{4}+2 \beta \kappa r^{2} \sin (\theta-\omega \tau)\left(1+e^{-\lambda \tau}\right) \\
\quad-\kappa^{2} \sin ^{2}(\theta-\omega \tau)\left(1+e^{-\lambda \tau}\right)^{2} .
\end{aligned}
$$

A Hopf bifurcation in the transverse direction can give rise to antiphase oscillations of the amplitude.
We start by considering the situation with instantaneous coupling. Then we shall proceed to follow the bifurcating events as we increase the delay. For the zero delay case we find in the transverse direction the eigenvalues

$$
\lambda=-r^{2}-2 \kappa \cos \theta \pm \sqrt{r^{4}+4 \beta \kappa \sin \theta-4 \kappa^{2} \sin ^{2} \theta} .
$$

Consequently, solutions which are saddle points or unstable foci can also exist without coupling delay in the transverse direction. On the contrary, for the parallel direction the modes (and antimodes) only become unstable when coupling strength and delay time are sufficiently large. If the coupling delay remains small, instabilities hence only occur in transverse direction.

When increasing the coupling delay, the difference in stability in transverse and parallel direction shrinks and in the long delay limit the stability of the modes is the same in both directions. Still, as we shall show next, a mode always undergoes a Hopf bifurcation first in the transverse direction. As a consequence, the isochronal oscillatory solution is always unstable.

Regarding the frequency of the emerging amplitude oscillations, for long delay times, we can show that Hopf bifurcations in the transverse direction lead to a period approximately twice the delay time,

$$
P \approx 2 \tau+\frac{2}{\kappa \sqrt{1+\beta^{2}} \cos (\arctan \beta-\omega \tau+\theta)} .
$$

Since amplitude oscillations are in antiphase with each other, the elements differ by approximately one delay time, also resembling the leader-laggard type of dynamics in delaycoupled semiconductor lasers.

\section{B. Antiphase amplitude oscillations}

To study the onset of the antiphase dynamics, it will be useful to directly compare the eigenvalues for both directions. To do so we unify characteristic equations (9) and (15) by rewriting them as follows:

$$
\lambda=-r^{2}-\kappa\left(1-e^{i \psi-\lambda \tau}\right) \cos (\omega \tau)+\sqrt{r^{4}-2 \beta \kappa r^{2} \sin (\omega \tau)\left(1-e^{i \psi-\lambda \tau}\right)-\kappa^{2} \sin ^{2}(\omega \tau)\left(1-e^{i \psi-\lambda \tau}\right)^{2}},
$$

where $\psi=2 \pi$ is used for the parallel direction and $\psi=\pi$ for the transverse direction. Although $\psi$ can only take a discrete number of values depending on the coupling topology, we will treat it here as a continuous variable. ${ }^{49}$ For the sake of simplicity in the next computations, we choose $\theta=0$ (no coupling phase) and $\Delta \phi=0$ (in-phase mode). The results can easily be generalized by replacing $(\omega \tau)$ by $(\omega \tau-\theta-\Delta \phi)$ in all equations. In the following we consider a long coupling delay $\tau$.

The rationale for demonstrating the instability of inphase amplitude oscillations lies in proving that a mode looses sooner its stability as a function of $\tau$ when the value of $\psi$ is smaller. To show this we proceed by assuming that for a certain set of parameters $\left(\kappa^{*}, \tau^{*}, \omega \tau^{*}\right)$, a mode undergoes a Hopf bifurcation for an angle $\psi^{*}$. By substituting in Eq. (18) the set of critical parameters, $\lambda=i \sigma$ and $\psi=\psi^{*}$, we obtain

$$
\begin{aligned}
i \sigma= & -r^{2}-\kappa^{*}\left(1-e^{i \psi^{*}-i \sigma \tau^{*}}\right) \cos \left(\omega \tau^{*}\right) \\
& +\left(r^{4}-2 \beta^{*} \kappa^{*} r^{2} \sin \left(\omega \tau^{*}\right)\left(1-e^{i \psi^{*}-i \sigma \tau^{*}}\right)\right. \\
& \left.-\kappa^{2} \sin ^{2}\left(\omega \tau^{*}\right)\left(1-e^{i \psi^{*}-i \sigma \tau^{*}}\right)^{2}\right)^{1 / 2},
\end{aligned}
$$


with $\sigma \tau=\psi^{*}+O(1 / \tau)$, from Eqs. (10) and (16). If we demonstrate that this mode is stable for $\psi>\psi^{*}$ and unstable for $\psi<\psi^{*}$ for the parameters $\left(\kappa^{*}, \tau^{*}, \omega \tau^{*}\right)$, we will have proven that amplitude instabilities always occur sooner in the transverse direction $\psi=\pi$ than in parallel direction $\psi=2 \pi$. This means that isochronal synchronization is always unstable in such systems.

We proceed then by considering a small deviation of the critical angle $\psi=\psi^{*}+\delta$, with $\delta \ll 1$, and check the sign of the real part of the new eigenvalue $i \sigma+\epsilon$. By the characteristic equation we obtain that

$$
\begin{aligned}
\epsilon \approx & \left(i \delta-\epsilon \tau^{*}\right) e^{i \psi^{*}-i \sigma \tau^{*}}\left(\kappa^{*} \cos \left(\omega \tau^{*}\right)\right. \\
& \left.+\frac{\beta^{*} \kappa^{*} r^{2} \sin \omega \tau^{*}+\kappa^{* 2} \sin ^{2}\left(\omega \tau^{*}\right)\left(1-e^{i \psi^{*}-i \sigma \tau^{*}}\right)}{r^{2}+i \sigma+\kappa^{*} \cos \omega \tau^{*}\left(1-e^{i \psi^{*}-i \sigma \tau^{*}}\right)}\right) .
\end{aligned}
$$

This expression for $\epsilon$ can be rewritten as

$$
\epsilon \approx i \delta \frac{A}{1+A \tau}
$$

with

$$
\begin{aligned}
A= & e^{i \psi^{*}-i \sigma \tau^{*}}\left(\kappa^{*} \cos \left(\omega \tau^{*}\right)\right. \\
& \left.+\frac{\beta^{*} \kappa^{*} r^{2} \sin \omega \tau^{*}+\kappa^{* 2} \sin ^{2}\left(\omega \tau^{*}\right)\left(1-e^{i \psi^{*}-i \sigma \tau^{*}}\right)}{r^{2}+i \sigma+\kappa^{*} \cos \omega \tau^{*}\left(1-e^{i \psi^{*}-i \sigma \tau^{*}}\right)}\right) .
\end{aligned}
$$

Since $e^{i \psi^{*}-i \sigma \tau^{*}} \approx 1$ and $\sigma \approx 0$ for long coupling delays, we obtain in first order of $1 / \tau$

$$
A=e^{i \psi^{*}-i \sigma \tau^{*}} \sqrt{1+\beta^{* 2}} \cos \left(\omega \tau^{*}-\arctan \beta^{*}\right) .
$$

From Eq. (20) we see that $\epsilon$ has negative real part if $\delta>0$ and $\operatorname{Im}(A)>0$. We start then by inspecting the sign of the imaginary part of $A$ from its factors in Eq. (22). From Eqs. (11) and (16) we deduce that $\psi^{*}-\sigma \tau>0$ is a small positive angle, meaning that the oscillation period is slightly longer than one delay time, in parallel direction, or slightly longer than two delay times, in transverse direction. The imaginary part of $e^{i \psi^{*}-i \sigma \tau^{*}}$ is hence always positive. The term $\cos \left(\omega \tau^{*}\right.$ $\left.-\arctan \beta^{*}\right)$ is positive for the modes and negative for the antimodes. Therefore, we deduce that $\operatorname{Im}(A)>0$ for the modes, and thus $\operatorname{Re}(\epsilon)<0$ if $\delta>0$ and vice versa.

To check the validity of our assumptions, we compute the imaginary part of $A$ from Eq. (21), without omitting any of the terms. As shown in Fig. 6, the condition $\operatorname{Im}(A)>0$ is fulfilled for the most of the modes, under the assumptions of $\sigma=O(1 / \tau)$ and $i \psi-i \sigma \tau=O(1 / \tau)$, including the Hopf bifurcation point for infinite delay. Hence, we can conclude that the transverse Hopf bifurcation will always precede the bifurcation in parallel direction.

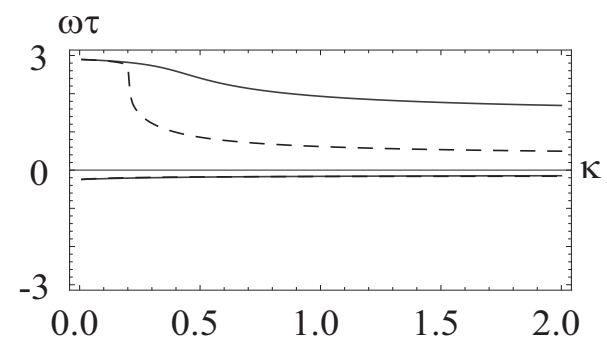

FIG. 6. The ellipse, parametrized by $\omega \tau$, is plotted vs coupling strength $\kappa$. The black lines mark the range of $\omega \tau$ for which the proof is valid $\operatorname{Im}(A)$ $>0)$. Between the dotted lines mark the modes are stable. We chose $\sigma \rightarrow 0, i \psi-i \sigma \tau=0.002$ and $\beta=4$.

\section{Chaotic dynamics of leader-laggard type}

We complement our analysis with numerical simulations of two mutually coupled oscillators with delay. For each simulation we initialized the oscillators with an amplitude $r_{k}=1$ and $\omega=\beta$ and the phases close to each other, in order to detect a stable zero-lag synchronized solution.

First, we have checked the influence of coupling phase and initial phases. The coupling phase $\theta$ can stabilize or destabilize a mode with constant amplitude, in a similar way as for noninvasively coupled oscillators; ${ }^{36}$ in the limit of weak coupling, for phase oscillators, this can easily be seen in stability criteria (13) and (14). This effect is only important for short coupling delays, when there exist only few modes and when most of them are stable. The coupling phase does not play a role for the occurring oscillation pattern of the amplitude. The initial phases determine the mode(s) which are involved in the dynamics. They are important when the amplitude is constant or periodic, but no longer in the chaotic regime.

The influence of the coupling delay is characterized by bifurcation plot as shown in Fig. 7. To better compare the mutually coupled case with the feedback system, we plot the bifurcation diagram against the total round-trip time, which for the mutually coupled elements amounts to twice the coupling delay. For small delays the two oscillators are synchronized in an in-phase mode with constant amplitude and showing the same behavior as for one oscillator with feedback. However, when the elements start oscillating around

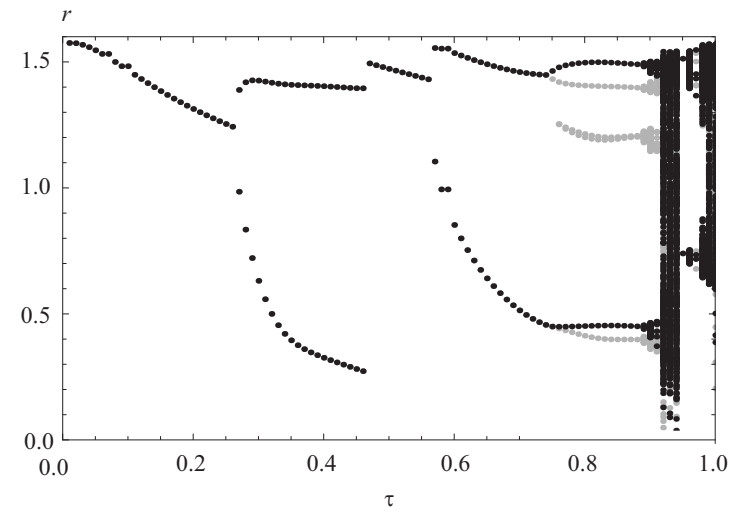

FIG. 7. The bifurcation diagram for two Stuart-Landau oscillators mutually coupled with delay. The extrema of the first oscillator are in black, those of the second one in gray. Parameters $\beta=4, \kappa=1.5$, and $\theta=0$. 


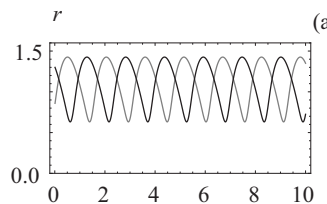

(a)

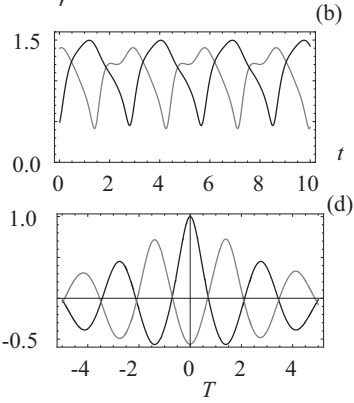

FIG. 8. Amplitude dynamics [(a)-(c)] and auto- and cross-correlation functions (d) of two delay-coupled Stuart-Landau oscillators. In (a)-(c) the black line represents the first oscillator and the gray line the second oscillator. In (d) the black curve shows the autocorrelation of one oscillator and the gray curve represents the cross-correlation between the oscillators, as a function of the time lag. (a) $\tau=0.3$, (b) $\tau=0.8$, and [(c) and (d) $\tau=0.93$. In all panels $\beta=4, \kappa=1.5$, and $\theta=0$.

this mode (at $\tau \approx 0.27$ ), the oscillations in amplitude are always out-of-phase, as plotted in Fig. 8(a). In contrast to the feedback system, the chaos does not develop further, but the system reaches a stable antiphase mode with constant amplitude $(\tau \approx 0.47)$. With increasing delay, new solutions emerge at the top of the ellipse. From characteristic equations (9) and (15), it can be deduced that a newborn mode, with a large constant amplitude, is stable. When increasing the coupling delay further, also this mode looses stability and antiphase oscillations emerge again $(\tau \approx 0.57)$.

Symmetry breaks for larger delay times; while the amplitudes oscillate in antiphase after the first Hopf bifurcation, they describe a different orbit (with the same period) when $(\tau \approx 0.76)$. In Fig. $8(b)$ it is shown how one oscillator describes an orbit with two maxima of amplitude per period, while the other oscillator has only one maximum. Further increasing the coupling delay, we enter in the chaotic regime where symmetry is restored and a generalized synchronization of leader-laggard type is observed [Figs. 8(c) and 8(d)]. As can be seen from the auto- and cross-correlation functions, the out-of-phase pattern is clearly still reflected in the dynamics.

We complete the analysis by investigating the influence of the coupling strength between the two oscillators. We performed a two-dimensional parameter scan in the $(\kappa, \tau)$-plane, and for each simulation, we classify the dynamical state of the two oscillators. The results are shown in Fig. 9. We can notice that the transitions induced by the delay are maintained for a range of coupling strengths, and therefore the picture of symmetry-transitions presented before can be considered generic to some extent. The stable amplitude region in between two oscillatory regions is due to the emergence of a stable antiphase mode, as discussed above with respect to the bifurcation plot (Fig. 7). In agreement with the theoretical arguments, the complete synchronization $\left(a_{1}(t)=a_{2}(t)\right)$ is only stable when the amplitude is constant. In other words when a mode looses stability and the system starts oscillating, these oscillations will always be in antiphase.

In our analysis and simulations, we did neither consider the influence of a detuning nor a noise source. We expect the steady state (constant amplitude) solutions to be stable to

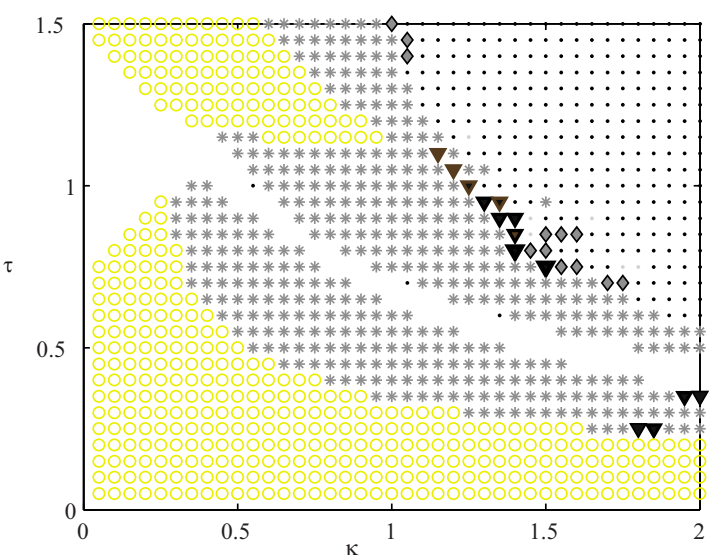

FIG. 9. (Color online) Dynamical behavior of two mutually delay-coupled Stuart-Landau oscillators for varying coupling delay $\tau$ and coupling strength $\kappa$. For the white region both amplitudes are constant. Gray crosses denote (out-of-phase) period 1 oscillations, diamonds denote that one of the two oscillators has undergone a period-doubling, and triangles represent different oscillations of the two elements, but with the same period. The black dots correspond to chaotic dynamics. When the oscillators are isochronously synchronized, a gray (yellow) circle is drawn. Parameters are chosen $\beta=4$ and $\theta=0$.

noise. A small detuning would cause small differences in phase and amplitude in the steady state regime and would affect the leader-laggard relation in the chaotic regime.

\section{STABILIZATION OF ZERO-LAG SYNCHRONIZATION BY A RELAY}

Next we investigate the situation in which two oscillators are coupled indirectly. In particular, we analyze a coupling through a third relay element. A schematic picture of the configuration is shown in Fig. 10. Notice that in such a setup the two oscillators always receive the same input from the relay, independent of whether the network is synchronized.

We consider here the most simple kind of relay, one in which the relay is a passive element that redistributes the signals of two oscillators. Thus, such an element would be the analog of a semitransparent mirror, feeding back a part of the signal to each oscillator and letting another part pass through to be received by the other oscillator. Accordingly, the equations that govern the dynamics are

$$
\dot{a}_{k}=a_{k}\left(1-\left|a_{k}\right|^{2}\right)+i \beta a_{k}\left|a_{k}\right|^{2}+\frac{\kappa e^{i \theta}}{2}\left(a_{k}(t-\tau)+a_{3-k}(t-\tau)\right)
$$

for $k=1,2$.

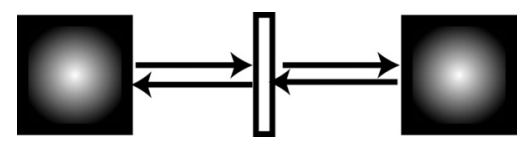

FIG. 10. Configuration of two oscillators coupled through a passive relay. 

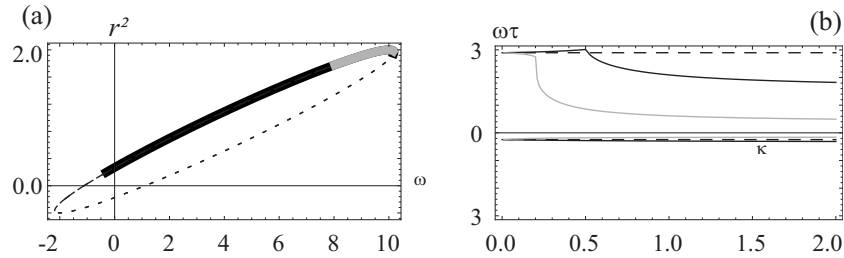

FIG. 11. (a) The picture shows the ellipse in the $\left(r^{2}, \omega\right)$-plane on which the in-phase stable amplitude solutions of system (23) are located. The gray part of the ellipse contains stable foci, while the black part contains foci which are stable in transverse direction but unstable in parallel direction. Unstable foci are located in the dashed part. The dotted part covers saddle points. $\kappa$ $=2$ and $\beta=4$. Long delay limit. (b) Stability of the modes for varying coupling strength. Portions of the ellipse (as parametrized by $\omega \tau-\theta$ ) which are stable are contained within the gray lines. Portions which are transversely stable are contained within the black lines. $\beta=4$. Long delay limit.

\section{A. Stability of the modes}

The in-phase modes of the system are the same as for one oscillator with feedback. In contrast, there is only one antiphase mode possible, $r_{k}=1, \omega=\beta$. This asymmetric solution is, already in the phase oscillator network, only stable for weak coupling strengths and short coupling delays. ${ }^{39}$

Just as in the case of direct mutual coupling, the stability of the in-phase modes along the synchronization manifold is given by Eq. (9). Nevertheless, the transverse stability of the (in-phase) modes dramatically increases due to the relay. Moreover, because the oscillators receive exactly the same input, the delayed terms cancel in transverse direction. Thus, we find a characteristic equation,

$$
\begin{aligned}
\lambda= & -r^{2}-\kappa \cos (\theta-\omega \tau) \\
& +\sqrt{r^{4}+2 \beta \kappa r^{2} \sin (\theta-\omega \tau)-\kappa^{2} \sin ^{2}(\theta-\omega \tau)},
\end{aligned}
$$

which is no longer transcendental. As a consequence, for sufficiently long delay times, a large part of the ellipse can become unstable in parallel direction and still remain stable in transverse direction (as can be seen in Fig. 11).

When the system does not have a stable amplitude, the linear stability of the synchronization manifold can be calculated by applying a small perturbation $A(t)$ in transverse direction. The dynamics of $A(t)$ is up to first order governed by the following time-dependent variational equation:

$$
\begin{aligned}
\dot{A}(t)= & \left(-r(t)^{2}-\kappa \cos (\theta+\phi(t-\tau)-\phi(t))+\left(r(t)^{4}\right.\right. \\
& +2 \beta \kappa r(t)^{2} \sin (\theta+\phi(t-\tau)-\phi(t)) \\
& \left.-\kappa^{2} \sin ^{2}(\theta+\phi(t-\tau)-\phi(t))\right)^{1 / 2} A(t) .
\end{aligned}
$$

Therefore, in periodic or chaotic regimes the transverse stability of the synchronization manifold does not depend explicitly on the time lag $\tau$. Chaos is hence more likely to develop within the parallel direction, which implies that the oscillators remain synchronized.

The same analysis can be extended for any network where two elements receive exactly the same input (then the delay terms cancel in the transverse characteristic equation). Other examples, as the two outer oscillators in a chain of three, or one oscillator with feedback injecting in another oscillator, are shown in Fig. 12. In these cases the dynamics within the synchronization manifold (for the outer elements

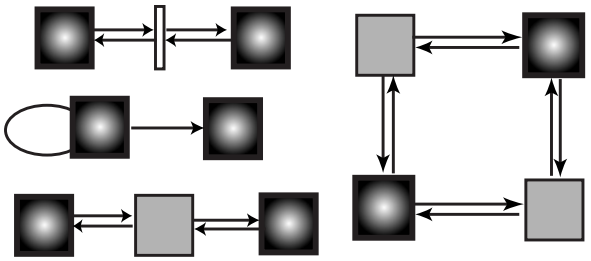

FIG. 12. A few examples of networks in which the similar nodes receive the same input and will hence be likely to synchronize.

of a chain), or the "transverse" direction (in the "masterslave" configuration) can be different, but transverse stability is identical.

\section{B. Complete chaos synchronization}

To check the synchronization properties when the amplitudes are no longer stable, we simulated numerically two Stuart-Landau oscillators coupled through a passive relay (or semitransparent mirror for optical devices), starting from the same initial conditions as before. We performed a scanning of the two-dimensional parameter $(\kappa-\tau)$ plane followed by the classification of the dynamical state. The results are presented in Fig. 13. For the vast majority of cases, after a variable transient the network synchronized identically. Only for short delays or small coupling strengths, antiphase solutions are possible. Once the amplitudes start oscillating, the oscillations are always in-phase. At the period-doubling bifurcations, the oscillators perform the same oscillatory orbit, but in antiphase with each other, but complete synchronization is restored once chaos develops.

Here we have considered only identical oscillators and identical parameters for the feedback and the delayed coupling, and we did not take any noise source into account. When the oscillators are noisy and slightly mismatched, we typically observe a bubbling behavior in the system.

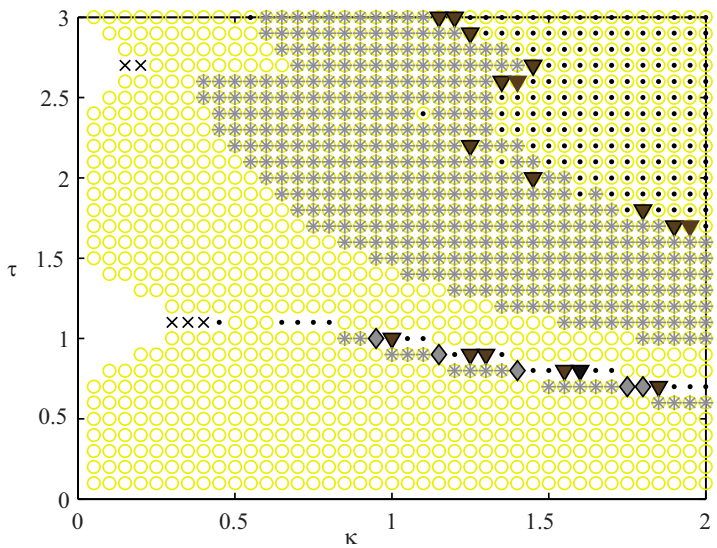

FIG. 13. (Color online) Dynamical behavior of two mutually delay-coupled Stuart-Landau oscillators with feedback for varying coupling delay $\tau$ and coupling strength $\kappa$. In the white region the amplitudes are constant, the gray crosses denote period 1 oscillations (mainly in-phase), the diamonds denote that the oscillators have undergone a period-doubling (and now oscillate out-of-phase), and the triangles represent different oscillations of the two elements, but with the same period. The black dots correspond to chaotic dynamics. When the oscillators are synchronized, a gray (yellow) circle is drawn. Parameters: $\beta=4$ and $\theta=0$. 


\section{CONCLUSIONS}

In this work we have compared the dynamics of one oscillator with delayed feedback, two mutually coupled oscillators, and two mutually coupled oscillators with feedback. Additionally, we have compared two levels of descriptions of the oscillators (with and without considering an amplitude) which allowed us to identify the variables responsible for various bifurcations such as symmetry transitions and synchronization phenomena.

We reviewed the dynamics of one Stuart-Landau oscillator with feedback. The delay induced multiple solutions with constant amplitude that can be distinguished in foci and saddle solutions. The feedback delay induced first oscillatory behavior and then chaos if the feedback strength was sufficiently strong. We also observed that an amplitude-phase coupling $\beta$ (the analog of the linewidth enhancement factor) is necessary to destabilize a mode.

When two oscillators are mutually coupled, in-phase and antiphase modes are possible. For weak coupling and low amplitude-phase coupling, the dynamics is well described by a Kuramoto system; the stability of in-phase and antiphase modes is then very comparable. However, when the coupling delay induces amplitude oscillations, only one type of oscillations is possible. This synchronization pattern does not relate to the phase difference $\Delta \phi$ of the oscillators, it originates from the antiphase oscillations induced in amplitude (and frequency). There is hence no connection between phaseshifted states in the Kuramoto model and time lags in the case of chaotic Stuart-Landau dynamics. The antiphase pattern arises naturally on the route to chaos; we prove analytically that these antiphase oscillations are due a Hopf bifurcation in transverse direction, while the mode is stable in parallel direction.

If we add delayed feedback (with same delay, strength, and phase as the coupling) to the oscillators, the isochronously synchronized solutions can be stabilized. Because the elements receive exactly the same input independent whether they are synchronized or not, the coupling delay disappears in the transverse characteristic equation. Consequently, the transverse direction is stablized and oscillations occur in parallel direction and are hence in-phase. We confirmed numerically that such a configuration is likely to show complete synchronization.

Current investigation is oriented to extend our results to more complex networks and characterizing the role played by coupling delays in synchronization patterns.

\section{ACKNOWLEDGMENTS}

We would like to thank T. Erneux, E. Schöll, S. Yanchuk, and P. Perlikowski for helpful discussions. O.D. acknowledges the Research Foundation Flanders (FWO-Vlaanderen) for a fellowship and for project support. This work was partially supported by the Interuniversity Attraction Poles program of the Belgian Science Policy Office, under Grant No. IAP VI-10 "photonics@be," by MICINN (Spain) under project DeCoDicA (Grant No. TEC200914101), and by the project PHOCUS (EU FET Open Grant No. 240763).
${ }^{1}$ S. Boccaletti, J. Kurths, G. Osipov, D. L. Valladares, and C. S. Zhou, Phys. Rep. 366, 1 (2002).

${ }^{2}$ A. Pikovsky, M. G. Rosenblum, and J. Kurths, Synchronization, A Universal Concept in Nonlinear Sciences (Cambridge University Press, Cambridge, 2001).

${ }^{3}$ W. Singer, Nature (London) 397, 391 (1999).

${ }^{4}$ F. Varela, J. Lachaux, E. Rodriguez, and J. Martinerie, Nat. Rev. Neurosci. 2, 229 (2001).

${ }^{5}$ H. G. Winful and L. Rahman, Phys. Rev. Lett. 65, 1575 (1990).

${ }^{6}$ M. Y. Kim, R. Roy, J. L. Aron, T. W. Carr, and I. B. Schwartz, Phys. Rev. Lett. 94, 088101 (2005).

${ }^{7}$ K. Wiesenfeld, P. Colet, and S. H. Strogatz, Phys. Rev. Lett. 76, 404 (1996).

${ }^{8}$ G. Filatrella, N. F. Pedersen, and K. Wiesenfeld, Phys. Rev. E 61, 2513 (2000).

${ }^{9}$ Z. Néda, E. Ravasz, Y. Brechet, T. Vicsek, and A. Barabasi, Nature (London) 403, 849 (2000).

${ }^{10}$ Y. Marchenko and V. Rubanik, Izv. Vyssh. Uchebn. Zaved., Radiofiz. 8 679 (1965).

${ }^{11}$ Y. Marchenko, Izv. Vyssh. Uchebn. Zaved., Radiofiz. 10, 1533 (1967).

${ }^{12}$ A. Kouda and S. Mori, IEEE Trans. Circuits Syst. CAS-28, 247 (1981).

${ }^{13}$ G. Orosz, R. E. Wilson, R. Szalai, and G. Stépan, Phys. Rev. E 80, 046205 (2009).

${ }^{14}$ M. K. Yeung and S. H. Strogatz, Phys. Rev. Lett. 82, 648 (1999).

${ }^{15}$ C. Masoller, A. Marti, and D. Zanette, Physica A 325, 186 (2003).

${ }^{16}$ T. Erneux, Applied Delay Differential Equations (Springer, New York, 2009).

${ }^{17}$ B. Balachandran, T. Kamár-Nagy, and D. Gilsinn, Delay Differential Equations, Recent Advances and New Directions (Springer, New York, 2009).

${ }^{18}$ W. Michiels and S.-I. Niculescu, Stability and Stabilization of Time-Delay Systems. An Eigenvalue Based Approach, Advances in Design and Control Vol. 12 (SIAM, Philadelphia, 2007).

${ }^{19}$ G. Stepan, Philos. Trans. R. Soc. London, Ser. A 367, 1059 (2009).

${ }^{20}$ W. Just, A. Pelster, M. Schanz and E. Schöll, Philos. Trans. R. Soc. London, Ser. A 368, 303 (2010).

${ }^{21}$ T. Heil, I. Fischer, W. Elsäßer, J. Mulet, and C. R. Mirasso, Phys. Rev. Lett. 86, 795 (2001).

${ }^{22}$ F. Rogister and J. Garcia-Ojalvo, Opt. Lett. 28, 1176 (2003).

${ }^{23}$ J. Mulet, C. R. Mirasso, T. Heil, and I. Fischer, J. Opt. Soc. Am. B 6, 97 (2004).

${ }^{24}$ J. K. White, M. Matus, and J. V. Moloney, Phys. Rev. E 65, 036229 (2002)

${ }^{25}$ S. Tang, R. Vicente, M. Chiang, C. R. Mirasso, and J. Liu, IEEE J. Sel. Top. Quantum Electron. 10, 936 (2004).

${ }^{26}$ A. Wagemakers, J. Buldu, and M. Sanjuan, Chaos 17, 023128 (2007).

${ }^{27}$ I. Fischer, R. Vicente, J. M. Buldú, M. Peil, C. R. Mirasso, M. C. Torrent, and J. García-Ojalvo, Phys. Rev. Lett. 97, 123902 (2006).

${ }^{28}$ E. Klein, N. Gross, M. Rosenbluh, W. Kinzel, L. Khaykovich, and I. Kanter, Phys. Rev. E 73, 066214 (2006).

${ }^{29}$ R. Vicente, S. Tang, J. Mulet, C. R. Mirasso, and J.-M. Liu, Phys. Rev. E 73, 047201 (2006).

${ }^{30}$ R. Vicente, L. Gollo, C. Mirasso, I. Fischer, and G. Pipa, Proc. Natl. Acad. Sci. U.S.A. 105, 17157 (2008).

${ }^{31}$ Y. Kuramoto, Int. J. Bifurcation Chaos Appl. Sci. Eng. 7, 789 (1997).

${ }^{32}$ H. Daido, Int. J. Bifurcation Chaos Appl. Sci. Eng. 7, 807 (1997).

${ }^{33}$ S. Wirkus and R. Rand, Nonlinear Dyn. 30, 205 (2002).

${ }^{34}$ D. V. Ramana Reddy, A. Sen, and G. L. Johnston, Phys. Rev. Lett. 85 3381 (2000).

${ }^{35}$ R. Dodla, A. Sen, and G. L. Johnston, Phys. Rev. E 69, 056217 (2004).

${ }^{36}$ C. U. Choe, T. Dahms, P. Hövel, and E. Schöll, Phys. Rev. E 81, 025205(R) (2010)

${ }^{37}$ D. Pieroux and P. Mandel, Phys. Rev. E 68, 036204 (2003).

${ }^{38}$ M. G. Earl and S. H. Strogatz, Phys. Rev. E 67, 036204 (2003).

${ }^{39}$ O. D’Huys, R. Vicente, T. Erneux, J. Danckaert, and I. Fischer, Chaos 18, 037116 (2008).

${ }^{40}$ S. Yanchuk, Math. Methods Appl. Sci. 28, 363 (2005).

${ }^{41}$ S. Yanchuk and P. Perlikowski, Phys. Rev. E 79, 046221 (2009).

${ }^{42}$ S. Yanchuk, M. Wolfrum, P. Hövel, and E. Schöll, Phys. Rev. E 74 026201 (2006).

${ }^{43}$ R. Lang and K. Kobayashi, IEEE J. Quantum Electron. 16, 347 (1980).

${ }^{44}$ A. M. Levine, G. H. M. van Tartwijk, D. Lenstra, and T. Erneux, Phys. Rev. A 52, R3436 (1995).

${ }^{45}$ T. Sano, Phys. Rev. A 50, 2719 (1994). 
${ }^{46}$ G. Van Tartwijk, A. Levine, and D. Lenstra, IEEE J. Sel. Top. Quantum Electron. 1, 466 (1995).

${ }^{47}$ R. Davidchack, Y. Lai, A. Gavrielides, and V. Kovanis, Phys. Lett. A 267, 350 (2000).

${ }^{48}$ H. G. Schuster and P. Wagner, Prog. Theor. Phys. 81, 939 (1989).
${ }^{49}$ The angle $\psi$ refers to the eigenvector $\left(1, e^{i \psi}\right)$ of the adjecancy matrix of the network. Since for a unidirectional ring, the eigenvectors are of the same form, this proof can be generalized. We obtain there that stability will break first for the smallest possible value of $\psi=2 \pi / N$ for $N$ the number of elements in the ring. 